\title{
CARACTERIZACIÓN ANATÓMICA Y CONDUCTIVIDAD HIDRÁULICA TEÓRICA DE LOS ELEMENTOS DE VASO DE VARIEDADES SILVESTRES Y CULTIVADAS DE NOPAL TUNERO (OPUNTIA)
}

\author{
Eulogio Pimienta-BarRios \\ Departamento de Ecología \\ Universidad de Guadalajara \\ Centro Universitario de Ciencias Biológicas y Agropecuarias \\ carretera a Nogales $\mathrm{km} 15.5$ \\ 45110 Zapopan, Jalisco, México \\ Sofía Loza Cornejo \\ Programa de Botánica \\ Colegio de Postgraduados \\ Carretera México-Texcoco km 35.5 \\ 56230 Montecillo, Texcoco \\ Edo. de México \\ Y \\ María Eugenia González del Castillo-Aranda \\ Departamento de Ecología \\ Universidad de Guadalajara \\ Centro Universitario de Ciencias Biológicas y Agropecuarias \\ carretera a Nogales $\mathrm{km} 15.5$ \\ 45110 Zapopan, Jalisco, México
}

\section{RESUMEN}

Se estudió la morfología y la conductancia hidráulica teórica de elementos de vaso del xilema en cladodios de especies y variedades cultivadas y silvestres de nopal (Opuntia spp.) colectados en las regiones semiáridas de los estados de Jalisco y Zacatecas, con el fin de investigar si las diferencias en la resistencia a la sequía que se observan comúnmente en el campo entre especies silvestres y cultivadas de Opuntia pueden estar relacionadas con divergencias en la densidad, la anatomía de los vasos y en la conductividad hidráulica. Adicionalmente se investigó si existen diferencias en el grado de especialización del xilema entre variedades cultivadas y silvestres. Nuestras observaciones mostraron que en la especie cultivada Opuntia ficus-indica (L.) Mill. (variedades amarilla montesa, picochulo, chapeada, pelón liso, burrona y cristalina), así como en las silvestres $O$. streptacantha Lem. (variedades cardón y charola), O. robusta Wendl. (variedades tapón y tapón de mayo) y $O$. cochinera Griff., es más frecuente encontrar elementos de vaso con engrosamientos escalariformes y alternos, que los provistos de engrosamientos helicoidales. Las 
plantas estudiadas no mostraron diferencias morfológicas que indicaran un mayor grado de especialización en las características morfológicas registradas en los elementos de vaso. Los caracteres morfológicos de los elementos de vaso, y los de conductividad hidráulica, no revelan diferencias que puedan ayudar a explicar la mayor resistencia a la sequía en las especies silvestres de nopal en comparación con las cultivadas.

Palabras clave: anatomía del xilema, conductividad hidráulica, evolución, Opuntia.

\begin{abstract}
The morphology and hydraulic conductance of xylem vessel members of cladodes of both wild and cultivated prickly pear species and varieties (Opuntia spp.) collected in the semiarid lands of the Mexican states of Jalisco and Zacatecas, were examined. We aimed to know if the differences in resistance to drought commonly observed in the field between wild and cultivated species of Opuntia might be related with density, anatomy of vessel members, and differences in their hydraulic conductance. In addition, we studied if there were differences in the degree of specialization of the xylem of both wild and cultivated populations. Our observations showed that in the cultivated species Opuntia ficus-indica (L.) Mill. (varieties amarilla montesa, picochulo, chapeada, pelón liso, burrona and cristalina), as well as in wild species $O$. streptacantha Lem. (varieties cardón and charola); $O$. robusta Wendl. (varieties tapón and tapón de mayo), and $O$. cochinera Griff., vessel elements with scalariform and alternate thickenings were more commonly observed than vessel elements with helical thickenings. The plants under study did not show morphological differences in the vessel elements that indicated a higher degree of specialization in any of them. Neither morphological characters nor hydraulic conductance values of vessel members revealed differences that contribute to explain the greater resistance to drought of wild species compared with cultivated species of prickly pear observed in the field.
\end{abstract}

Key words: evolution, hydraulic conductance, Opuntia, xylem anatomy.

\title{
INTRODUCCIÓN
}

En las regiones áridas y semiáridas de México diferentes factores limitan el crecimiento de las plantas. La escasez de agua es el principal elemento restrictivo, sin embargo, otras características ambientales como vientos secos, y cambios bruscos en la temperatura, tanto diurna como estacional, agudizan la falta de agua (Gibson, 1996; Smith et al., 1997).

La evolución de los miembros del subgénero Opuntia en este tipo de ambientes ha conducido a que diferentes especies desarrollen características morfológicas, anatómicas, fisiológicas y bioquímicas que les permiten adaptarse a tales condiciones adversas (Gibson y Nobel, 1986; Nobel, 1995; Gibson, 1996; Wickens, 1998). Diversos autores consideran que las características anatómicas de la epidermis, como densidad baja de estomas y cutícula gruesa, han tenido una función importante en la adaptación de los nopales a la aridez (Conde, 1975; Mauseth, 1984; Gibson y Nobel, 1986; Pimienta Barrios et al., 1993; Nobel, 1994; Nobel, 1995; Gibson, 1996; Wickens, 1998). En contraste con los estudios realizados a nivel de la epidermis, menos atención se ha prestado a las modificaciones que presenta el xilema en respuesta a la sequía (Conde, 1975; Gibson, 1977; 1978; Gibson, 1996; Linton y Nobel, 1999). Además, pocas investigaciones se han 
llevado a cabo sobre adaptaciones morfológicas a la aridez en especies de Opuntia en el sur del Desierto Chihuahuense, en donde se encuentra la variación más amplia de miembros de este género en condición cultivada y silvestre (Bravo-Hollis, 1978; PimientaBarrios, 1994).

En el campo, es común observar que las especies silvestres de nopal presenten una mayor resistencia a la sequía que las cultivadas (Pimienta-Barrios, 1990). Mientras en estas últimas la escasez de agua puede ocasionar daños severos e incluso la muerte de algunos individuos, las plantas espontáneas por el contrario, muestran en menor grado tales efectos (Pimienta-Barrios et al., 2002). Por ello, suponemos que las especies silvestres de nopal poseen adaptaciones estructurales y fisiológicas que facilitan esta resistencia a la sequía, particularmente en el xilema, como se ha encontrado en otras plantas que se desarrollan en ambientes áridos en California y en el Desierto del Negev (Gibson, 1996).

El xilema, además de la función de soporte mecánico, tiene también un papel importante en la regulación de la economía hídrica (Aston y Lawlor, 1979; Nobel, 1991). Los vasos estrechos son considerados como una ventaja adaptativa en hábitats secos, ya que son menos vulnerables a la cavitación (Carlquist, 1988; Yang y Tyree, 1992; Gibson, 1986; Mauseth y Plemons-Rodríguez, 1998). Por el contrario, los vasos anchos que permiten tasas mayores de flujo de agua, son más vulnerables a la cavitación por sequía (Yang y Tyree, 1992; Gibson, 1996), por lo que su presencia podría representar una desventaja para las plantas que crecen en ambientes que experimentan frecuentemente estrés por la escasez de agua (Gibson, 1986). En este trabajo postulamos la hipótesis de que las especies silvestres de nopal han desarrollado en el xilema modificaciones estructurales y funcionales, particularmente en los elementos de vaso que, en colaboración con otras adaptaciones fisiológicas, contribuyen a que éstas sean más resistentes a la sequía.

El objetivo de la presente contribución es determinar si existen diferencias en la morfología, densidad, tamaño, diámetro y conductividad hidráulica de los elementos de vaso entre la especie cultivada Opuntia ficus-indica (L.) Mill., y las especies silvestres $O$. streptacantha Lem., O. robusta Wendl. y O. cochinera Griff., consideradas como las más importantes en el Altiplano Potosino-Zacatecano (Pimienta-Barrios, 1994), que ayuden a explicar las diferencias en la resistencia a la sequía entre las plantas silvestres y cultivadas de nopal.

\section{MATERIALES Y MÉTODOS}

Se utilizaron cladodios maduros (2-3 años de edad) colectados en poblaciones silvestres y cultivadas de nopal en tres localidades de los estados de Zacatecas y Jalisco (Cuadro 1). En cada sitio se tomaron muestras de la parte media de cladodios basales de dos a tres individuos de cada especie y/o variedad presente. Los fragmentos extraídos con un sacabocados de $7.8 \mathrm{~mm}$ de diámetro se conservaron en formaldehido: ácido acético: alcohol (FAA, 10:5:85 en volumen) (Jensen, 1962) para su posterior procesamiento en laboratorio.

Para la obtención de material disociado se colocaron trozos de cladodios en viales con solución de Franklin (Berlyn y Miksche, 1976), los cuales se mantuvieron en una 
estufa a $60^{\circ} \mathrm{C}$ hasta que el tejido se observó blando y traslúcido. Posteriormente se le lavó y tiñó con safranina $(5 \%)$ y se hicieron preparaciones semipermanentes con glicerogelatina.

Cuadro 1. Especies y variedades cultivadas (C) y silvestres (S) de nopal tunero (Opuntia spp.) estudiadas en este trabajo.

\begin{tabular}{|l|l|c|l|}
\hline Variedad & Especie & Tipo de población & Localidad de colecta \\
\hline Amarilla montesa & Opuntia ficus-indica & C & La Palma, Zac. \\
Burrona & C & Ojuelos, Jal. \\
Cristalina & Cpuntia ficus-indica & C & Los Alpes, Zac. \\
Chapeada & Opuntia ficus-indica & C & La Palma, Zac. \\
Pelón liso & Opuntia ficus-indica & C & Ojuelos, Jal. \\
Picochulo & Opuntia ficus-indica & S & Ojuelos, Jal. \\
Cardón & Opuntia ficus-indica & S & La Palma, Zac. \\
Cochinera & Opuntia streptacantha & S & Los Alpes, Zac. \\
Charola & Opuntia cochinera & Ojuelos, Jal. \\
Tapón & Opuntia streptacantha & S & Ojuelos, Jal. \\
Tapón de mayo & Opuntia robusta & Opuntia robusta &
\end{tabular}

Los caracteres evaluados en el material disociado fueron: tipo de engrosamientos de la pared celular secundaria; tipo y orientación de la placa de perforación y longitud de los elementos de vaso. La descripción de los caracteres se hizo de acuerdo con Esau (1976). Para cada individuo se midieron 50 elementos de vaso utilizando el micrómetro del ocular de un microscopio Zeiss.

En los trozos de material fijado se hicieron cortes transversales del xilema secundario (aproximadamente $50 \mathrm{~mm}^{2}$ ) con la ayuda de una navaja de rasurar, se tiñeron con azul de toluidina (0.5\%; Berlyn y Miksche, 1976) durante 5 minutos y se observaron en un microscopio Zeiss. En estos cortes, en la parte externa de cada haz vascular, se midieron los siguientes caracteres: diámetro interno de los elementos de vaso (40x) y número de vasos por milímetro cuadrado, mismo que se determinó contando los vasos en forma individual en 25 campos (20x) para cada individuo (Gibson, 1978). La medición del diámetro se efectuó en corte transversal, en 220 a 460 elementos de vaso por cada entidad considerada.

Los valores obtenidos fueron utilizados para el cálculo de conductividad hidráulica teórica relativa $\left(\mathrm{K}_{\mathrm{h}}, \mathrm{m}^{4} \mathrm{MPa}^{-1} \mathrm{~s}^{-1}\right)$ mediante la siguiente ecuación (Gibson et al., 1984; Ewers y Fisher, 1989):

$$
\mathrm{K}_{\mathrm{h}}=\frac{\pi \sum \mathrm{d}_{\mathrm{i}}^{4}}{128 \eta}
$$

donde $\eta$ es la viscosidad dinámica del fluido (1.002 x 10-3 Pa.s; Nobel, 1991) y $\mathrm{d}_{\mathrm{i}}$ es el diámetro interno del vaso en metros. 
Para cada entidad estudiada se obtuvieron los valores de la media para las variables densidad, longitud y diámetro de los elementos de vaso. Para determinar diferencias estadísticas en el número de vasos por milímetro cuadrado y la longitud de los elementos de vaso entre las variedades, se aplicó un análisis de varianza seguido de una prueba de comparaciones múltiple de Duncan. En el caso del diámetro interno de los vasos, se utilizó la prueba de Kruskal-Wallis seguida del método de Dunn para evaluar diferencias entre las entidades. Se obtuvieron las medias de todas las variables medidas para plantas silvestres y cultivadas, y la diferencia entre estos valores se evaluó mediante una prueba t de Student, excepto en el caso de la conductividad hidráulica teórica, en que se empleó el procedimiento de Mann-Whitney (Zar, 1996).

\section{RESULTADOS Y DISCUSIÓN}

Pared celular de los elementos de vaso

Del total de los elementos de vaso observados, 35\% presentaron engrosamientos de tipo escalariforme, $36 \%$ de tipo alterno y $29 \%$ se caracterizó por la presencia de pared secundaria helicoidal (Cuadro 2). En todas las variedades se observó la presencia de placas de perforación simple. Los tipos más comunes de disposición de la placa de perforación fueron: transversal (53\%) y oblicua en transición a transversal (32\%); los elementos de vaso con disposición oblicua y lateral de la placa de perforación fueron menos frecuentes: $9 \%$ y $4 \%$ respectivamente (ver Cuadro 2). No se observaron diferencias estadísticas significativas entre los tipos de engrosamientos y disposición de la placa de perforación de los elementos de vaso entre las plantas silvestres y cultivadas $(P>0.1)$. Ambas se caracterizan por presentar engrosamientos escalariformes y alternos con placa de perforación simple más comúnmente transversal, lo cual coincide con las observaciones de Gibson $(1977,1978)$ para otras especies de Opuntia.

La presencia de engrosamientos escalariformes y alternos es una característica especializada para la familia Cactaceae (Preston, 1901); sin embargo, en el presente trabajo se observó que un porcentaje importante de los elementos de vaso tienen pared secundaria helicoidal, propiedad común del xilema primario (Esau, 1976). Carlquist (1962) señala que ciertos caracteres del xilema primario pueden permanecer en el xilema secundario (juvenilismo), lo cual cabe atribuir a la lenta acumulación de la pared secundaria en los elementos de vaso del xilema, como resultado de las condiciones de escasez de agua. Este fenómeno de pedomorfosis ha sido observado también en diversos miembros de la subfamilia Cactoideae (Gibson, 1973) y podría ser el caso de las especies y variedades de Opuntia aquí estudiadas. Los elementos de vaso con pared helicoidal proporcionan elasticidad al sistema vascular (Preston, 1901) y ayudan a mantener la integridad del flujo de agua dentro de los vasos durante periodos de sequía (Gibson, 1996). 
Cuadro 2. Caracteres anatómicos cualitativos de los elementos de vaso en variedades cultivadas (C) y silvestres (S) de nopal tunero (Opuntia spp.). Helicoidal $(H)$, escalariforme (E), alterna (A), oblicua $(\mathrm{O})$, oblicua en transición a transversal $(\mathrm{O} / \mathrm{T})$, transversal $(\mathrm{T})$, lateral $(\mathrm{L})$.

\begin{tabular}{|l|r|r|r|r|r|r|r|}
\hline \multirow{2}{*}{ Variedad } & \multicolumn{3}{|c|}{$\begin{array}{c}\text { Engrosamientos de la } \\
\text { pared celular (\%) }\end{array}$} & \multicolumn{3}{c|}{$\begin{array}{c}\text { Disposición de la placa } \\
\text { de perforación (\%) }\end{array}$} \\
\cline { 2 - 8 } & H & E & A & O & O/T & T & L \\
\hline Amarilla montesa (C) & 41 & 41 & 18 & 10 & 30 & 52 & 0 \\
Burrona (C) & 38 & 43 & 19 & 21 & 36 & 38 & 5 \\
Cristalina (C) & 42 & 51 & 7 & 7 & 29 & 61 & 3 \\
Chapeada (C) & 13 & 41 & 46 & 17 & 27 & 50 & 6 \\
Pelón liso (C) & 12 & 22 & 66 & 6 & 42 & 50 & 2 \\
Picochulo (C) & 17 & 20 & 63 & 11 & 25 & 64 & 0 \\
Cardón (S) & 18 & 32 & 50 & 6 & 31 & 60 & 3 \\
Cochinera (S) & 15 & 38 & 47 & 2 & 42 & 52 & 4 \\
Charola (S) & 50 & 29 & 21 & 4 & 30 & 61 & 5 \\
Tapón (S) & 23 & 20 & 57 & 12 & 37 & 38 & 13 \\
Tapón de mayo (S) & 47 & 48 & 5 & 7 & 27 & 60 & 6 \\
\hline
\end{tabular}

Longitud de elementos de vaso

Los elementos de vaso en las entidades de nopal estudiadas tienen una longitud promedio que varía entre 168 y $252 \mu \mathrm{m}$. No se observaron diferencias significativas $(P=0.38)$ entre las plantas silvestres y las cultivadas $(223.7 \pm 30.9 \mu \mathrm{m}$ y $206.8 \pm 28.6 \mu \mathrm{m}$, respectivamente; ver Cuadro 3). Los elementos de vaso con mayor longitud se registraron en tres variedades cultivadas de $O$. ficus-indica: "amarilla montesa", "burrona" y "pelón liso". En general, la longitud de los elementos de vaso en las entidades estudiadas es similar al intervalo (125 a $242 \mu \mathrm{m})$ que menciona Gibson (1978) para especies de nopales que producen frutos comestibles.

Diámetro de elementos de vaso

El diámetro de vasos en las especies y variedades estudiadas varía entre 29 y $50 \mu \mathrm{m}$. Al igual que para la longitud de elementos de vaso, no se observaron diferencias significativas entre plantas silvestres y cultivadas $(P=0.83)$. El diámetro promedio de los vasos fue mayor en las cultivadas $(38.2 \pm 6.9 \mu \mathrm{m})$ que en las silvestres $(37 \pm 8.0 \mu \mathrm{m})$. "Amarilla montesa" es una de las variedades cultivadas que se distinguió por los vasos más anchos (47 $\mu \mathrm{m})$, seguida por "picochulo", que también es cultivada (46 $\mu \mathrm{m})$. Ambas se caracterizan por su mayor tasa de crecimiento en el campo (Pimienta-Barrios, 1994), se agrupan dentro del conjunto de variedades conocidas como "amarillas", y presentan una amplia distribución geográfica en condición cultivada y silvestre en el mundo (Barbera, 1995; Pimienta-Barrios y Muñoz-Urias, 1995). El menor diámetro de vasos se encontró en la variedad silvestre "charola" (29 $\mu \mathrm{m})$ (Cuadro 3). El diámetro de vasos de 
las variedades estudiadas es similar al observado por Conde (1975) para dos especies silvestres de nopal (Opuntia lindheimeri y O. phaeacantha), pero es mayor que el de las especies de Opuntia con tallo cilíndrico (Gibson, 1977), ya que en éstas el diámetro de vasos varía de 16 a $80 \mu \mathrm{m}$.

Cuadro 3. Longitud promedio, diámetro promedio, densidad promedio y conductividad hidráulica teórica relativa de los elementos de vaso en variedades cultivadas (C) y silvestres (S) de nopal tunero (Opuntia spp.). Las medias de longitud, diámetro interno y número de vasos agrupadas con la misma letra dentro de columnas, no difieren estadísticamente.

\begin{tabular}{|l|c|c|c|c|}
\hline Variedad & $\begin{array}{c}\text { Longitud } \\
(\mu \mathrm{m})\end{array}$ & $\begin{array}{c}\text { Diámetro interno } \\
\mathrm{d}_{\mathrm{i}}(\mu \mathrm{m})\end{array}$ & $\begin{array}{c}\text { No vasos por } \\
\mathrm{mm}^{-2}\end{array}$ & $\begin{array}{c}\text { Conductividad } \\
\text { hidráulica teórica } \\
\mathrm{K}_{\mathrm{h}} \\
\left(10^{-14} \mathrm{~m}^{4} \mathrm{Mpa}^{-1} \mathrm{~s}^{-1}\right)\end{array}$ \\
\hline Amarilla montesa (C) & $252 \mathrm{a}$ & $46.7 \mathrm{a}$ & $5 \mathrm{ef}$ & 7.04 \\
Burrona (C) & $244 \mathrm{ab}$ & $35.0 \mathrm{bcd}$ & $6 \mathrm{de}$ & 1.55 \\
Cristalina (C) & $168 \mathrm{e}$ & $32.3 \mathrm{cde}$ & $4 \mathrm{f}$ & 0.96 \\
Chapeada (C) & $212 \mathrm{~cd}$ & $37.5 \mathrm{~b}$ & $9 \mathrm{a}$ & 3.99 \\
Pelón liso (C) & $242 \mathrm{ab}$ & $30.8 \mathrm{e}$ & $5 \mathrm{ef}$ & 0.78 \\
Picochulo (C) & $224 \mathrm{bc}$ & $46.5 \mathrm{a}$ & $7 \mathrm{c}$ & 5.46 \\
Cardón (S) & $191 \mathrm{~d}$ & $31.7 \mathrm{de}$ & $8 \mathrm{~b}$ & 1.67 \\
Cochinera (S) & $246 \mathrm{a}$ & $38.2 \mathrm{~b}$ & $4 \mathrm{f}$ & 2.10 \\
Charola (S) & $209 \mathrm{~cd}$ & $29.1 \mathrm{e}$ & $6 \mathrm{cde}$ & 0.69 \\
Tapón (S) & $218 \mathrm{c}$ & $36.5 \mathrm{~b}$ & $8 \mathrm{~b}$ & 2.95 \\
Tapón de mayo (S) & $170 \mathrm{e}$ & $35.3 \mathrm{bc}$ & $6 \mathrm{~cd}$ & 2.18 \\
\hline
\end{tabular}

Número de vasos por $\mathrm{mm}^{-2}$

En las variedades estudiadas, el número de vasos por $\mathrm{mm}^{-2}$ varía de 4 a 9 . No se observaron diferencias significativas en el promedio de esta variable entre las plantas cultivadas y las silvestres $\left(6.0 \pm 1.8\right.$ y $6.7 \pm 1.3$ vasos por $\mathrm{mm}^{-2}$, respectivamente, $\left.\mathrm{P}=0.72\right)$. La variedad cultivada "chapeada" muestra el valor más alto (9 vasos por $\mathrm{mm}^{-2}$ ); este valor es estadísticamente superior al registrado en el resto de las entidades estudiadas. Le siguen las variedades silvestres "cardón" (O. streptacantha) y "tapón" (O. robusta), ambas con 8 vasos por $\mathrm{mm}^{-2}$ (Cuadro 3).

Conductividad hidráulica teórica relativa $\left(\mathrm{K}_{\mathrm{h}}\right)$

No se encontraron diferencias estadísticas significativas en la conductividad hidráulica teórica del xilema entre las plantas cultivadas y silvestres objeto de este estudio $(\mathrm{P}=0.66)$, cuyas medias respectivas fueron $3.3 \times 10^{-14} \pm 2.6 \times 10^{-14}$ y $1.9 \times 10^{-14} \pm 8.3 \times$ $10^{-15}$. Los valores más altos se observaron en las tres variedades de la especie cultivada O. ficus-indica: "amarilla montesa", "picochulo" y "chapeada". La variedad "amarilla 
montesa" presenta una conductividad hidráulica que es aproximadamente diez veces mayor que la de la planta silvestre 0 . streptacantha var. "charola" (Cuadro 3). La diferencia se debe a que esta última posee una gran proporción de vasos de diámetro estrecho $(<40 \mu \mathrm{m})$, que contribuyen muy poco a la conductividad hidráulica.

De acuerdo con nuestra hipótesis, era de esperar que en las especies silvestres de Opuntia, los elementos de vaso presentaran modificaciones favorables para enfrentar las condiciones adversas de la sequía, como es la presencia de vasos de menor diámetro, ya que éstos son menos propensos al embolismo (Gibson, 1986; Yang y Tyree, 1992). Sin embargo, no se registraron diferencias en el diámetro de los vasos, ni tampoco en su densidad, longitud y tasas de conductividad hidráulica que ayuden a explicar la mayor resistencia a la aridez en las variedades silvestres. Las observaciones realizadas en otras especies leñosas del desierto de California han revelado que la presencia de un alto número de elementos de vaso estrechos es una estrategia alternativa para permitir que continúe la conducción de agua, aun cuando muchos de los vasos sean afectados por la cavitación (Gibson, 1996). Una tendencia similar se reveló en un estudio sobre las adaptaciones de la epidermis en un número amplio de variedades silvestres y cultivadas de nopal, que incluye a las plantas evaluadas en este trabajo (Pimienta-Barrios et al., 1993). En dicho estudio se encontró que los valores más altos de densidad de estomas (\$34 estomas por $\mathrm{mm}^{-2}$ ) se registraron en dos de las variedades silvestres consideradas aquí ("tapón" y "cardón"), mientras que dos de las variedades cultivadas ("pelón liso" y "burrona"), presentaron las densidades más bajas (\#17 estomas por $\mathrm{mm}^{-2}$ ). Por el contrario, el mayor grosor de la cutícula $(\$ 43 \mathrm{~mm})$ se encontró en dos de las variedades silvestres ("tapón" y "cardón"), y en una variedad cultivada: "amarilla huesona" (O. ficusindica). Aparentemente, no hay una relación consistente entre los atributos epidermales, o de elementos de vaso del xilema, y la resistencia y tolerancia a la escasez de agua en Opuntia.

Nuestros resultados son consistentes con otros estudios que incluyen especies de diversas líneas evolutivas dentro de la familia Cactaceae, en los que se encontró que especies adaptadas a hábitats áridos no presentan vasos de menor diámetro, o menores superficies de conducción que las plantas provenientes de ambientes húmedos (Gibson, 1973; Mauseth y Plemons-Rodríguez, 1998). Sin embargo, las diferencias en el diámetro y en la conductividad hidráulica de los elementos de vaso sí se encuentran relacionadas con otras características del desarrollo vegetal, como es el tamaño y el hábito de la planta (Gibson, 1973; Mauseth, 1993, Mauseth y Plemons-Rodríguez, 1998). Los resultados de este estudio refuerzan la observación de que los mecanismos morfogenéticos que controlan el desarrollo de los caracteres anatómicos del xilema en las cactáceas son muy estables y no responden a cambios de corto plazo en las condiciones ambientales (Arnold y Mauseth, 1999).

Lo anterior apoya la observación de que la mayor resistencia de las poblaciones silvestres a la sequía está relacionada con mecanismos fisiológicos de aclimatación a la escasez de agua, como es la fijación nocturna de $\mathrm{CO}_{2}$ (Nobel, 1994; Nobel, 1995). Este tipo de modificación fotosintética resulta reforzada por otras estrategias que contribuyen a la evasión y resistencia a la sequía en especies silvestres de Opuntia, entre las que destaca una mayor asimilación de $\mathrm{CO}_{2}$ al final de la noche, cuando la humedad relativa es más alta, y una mayor plasticidad fotosintética cuando aumenta la disponibilidad de 
agua en el ambiente (Pimienta-Barrios et al., 2002). Tal plasticidad fisiológica en respuesta a la disponibilidad de agua es común en las crasuláceas, plantas con metabolismo ácido, y aparentemente juega un papel importante en la resistencia a la sequía (Cushman, 2001).

\section{CONCLUSIONES}

1. El estudio anatómico de los elementos de vaso del xilema en las especies de Opuntia subgénero Opuntia consideradas en este trabajo, ha permitido verificar que los vasos de xilema son especializados, ya que las punteaduras intervasculares son predominantemente escalariformes y alternas.

2. El número y el diámetro de los vasos, así como los valores de conductividad hidráulica no mostraron diferencias estadísticas entre las variedades silvestres y las cultivadas de nopal, que ayuden a explicar las discrepancias en la resistencia a la sequía que se observan en condiciones naturales.

\section{AGRADECIMIENTOS}

Agradecemos a la Dra. Teresa Terrazas, del Colegio de Postgraduados de Montecillos, por las sugerencias y opiniones para la elaboración del presente artículo. Se agradecen también las valiosas observaciones de los árbitros, que fueron fundamentales para mejorar la calidad del artículo.

\section{LITERATURA CITADA}

Arnold, D. H. y J. D. Mauseth. 1999. Effects of environmental factors on development of wood. Amer. J. Bot. 86: 367-391.

Aston, M. J. y D. W. Lawlor. 1979. The relationship between transpiration, root water uptake and leaf water potential. J. Exp. Bot. 30: 169-181.

Barbera, G. 1995. History, economic and agro-ecological importance. In: Barbera, G., P. Inglese y E. Pimienta-Barrios (eds.). Agro-ecology, cultivation and uses of cactus pear. Food and Agriculture Organization. Roma. pp. 1-11.

Berlyn, G. P. y J. P. Miksche. 1976. Botanical microtechnique and cytochemistry. lowa State University Press. Ames. $326 \mathrm{pp}$.

Bravo-Hollis, H. 1978. Las cactáceas de México. Universidad Nacional Autónoma de México. México, D. F. Vol. 1. 743 pp.

Carlquist, S. 1962. A theory of paedomorphosis in dicotyledons woods. Phytomorphology 12: 30-45.

Carlquist, S. 1988. Near-vessellessness in Ephedra and its significance. Amer. J. Bot. 79: 660-672.

Conde, F. L. 1975. Anatomical comparisons of five species of Opuntia (Cactaceae). Ann. Miss. Bot. Garden 62: 425-473.

Cushman, J. C. 2001. Crassulacean acid metabolism. A plastic photosynthetic adaptation to arid environments. Plant Physiol. 127: 1439-1448.

Esau, K. 1976. Anatomía vegetal. Ediciones Omega. Barcelona. 779 pp.

Ewers, F. W. y J. B. Fisher. 1989. Techniques for measuring vessel lengths and diameters in stems of woody plants. Amer. J. Bot. 76: 645-656. 
Gibson, A. C. 1973. Comparative anatomy of secondary xylem in Cactoideae (Cactaceae). Biotropica 5: $29-65$.

Gibson, A. C. 1977. Wood anatomy of opuntias with cylindrical to globular stems. Bot. Gaz. 138: 334-351.

Gibson, A. C. 1978. Woody anatomy of platyopuntias. Aliso 9: 279-307.

Gibson, A. C. 1996. Structure-function relations of warm desert plants. Springer-Verlag. Heidelberg. $215 \mathrm{pp}$.

Gibson, A. C. y P. S. Nobel. 1986. The cactus primer. Harvard University Press. Cambridge, Mass. $286 \mathrm{pp}$.

Gibson, A. C., H. W. Calkin y P.S. Nobel. 1984. Xylem anatomy, water flow and hydraulic conductance in the fern Cyrtomium falcatum. Amer. J. Bot. 71: 564-574.

Jensen, W. A. 1962. Botanical histochemistry. W. H. Freeman and Co. San Francisco. 260 pp.

Linton, M. J. y P. S. Nobel. 1999. Loss of water transport capacity due to xylem cavitation in roots of two CAM succulents. Amer. J. Bot. 86: 1538-1543.

Mauseth, J. D. 1984. Introduction to cactus anatomy (Part 7). Epidermis. Cactus Succ. J. 56: 33-37.

Mauseth, J. D. 1993. Water-storing and cavitation-preventing adaptations in wood of cacti. Ann. Bot. 72: 81-89.

Mauseth, J. D. y J. Plemons-Rodríguez. 1998. Evolution of extreme xeromorphic characters in wood: a study of nine evolutionary lines in Cactaceae. Amer. J. Bot. 85: 209-218.

Nobel, P. S. 1991. Physicochemical and environmental plant physiology. Academic Press. San Diego. $635 \mathrm{pp}$.

Nobel, P. S. 1994. Remarkable agaves and cacti. Oxford University Press. Nueva York. 166 pp.

Nobel, P. S. 1995. Environmental biology. In: Barbera, G., P. Inglese y E. Pimienta-Barrios (eds.). Agro-ecology, cultivation and uses of cactus pear. Food and Agricultural Organization. Roma. pp. 36-48.

Pimienta-Barrios, E. 1990. El nopal tunero. Serie Libros Tiempos de Ciencia. Universidad de Guadalajara. Guadalajara. 246 pp.

Pimienta-Barrios, E. 1994. Prickly pear (Opuntia spp.): a valuable fruit crop for the semi-arid lands of Mexico. J. Arid Environ. 28: 1-11.

Pimienta-Barrios, E. y A. Muñoz-Urias. 1995. Domestication of opuntias and cultivated varieties. In: Barbera, G., P. Inglese y E. Pimienta-Barrios (eds.). Agro-ecology, cultivation and uses of cactus pear. Food and Agriculture Organization. Roma. pp. 58-63.

Pimienta-Barrios, E., M. Loera-Quezada y L. O. López-Amezcua. 1993. Estudio anatómico comparativo en colectas del subgénero Opuntia. Agrociencia serie Fitociencia 4(3): 7-21.

Pimienta-Barrios, E., M. E. González del Castillo-Aranda y P. S. Nobel. 2002. Ecophysiology of a wild platyopuntia exposed to prolonged drought. Env. Exp. Bot. 47: 77-86.

Preston, C. E. 1901. Structural studies on southwestern Cactaceae. Bot. Gaz. 32: 35-55.

Smith, D. S., R. K. Monson y J. E. Anderson. 1997. Physiological ecology of North American desert plants. Springer-Verlag. Heidelberg. $286 \mathrm{pp}$.

Yang, S. y M. T. Tyree. 1992. A theoretical model of hydraulic conductivity recovery from embolism with comparison to experimental data on Acer saccharum. Plant Cell Environ. 15: 633-643.

Wickens, E. G. 1998. Ecophysiology of economic plants in arid and semi-arid lands. Springer-Verlag. Heidelberg. 343 pp.

Zar, J. H. 1996. Biostatistical analysis. 3a. ed. Prentice Hall. Upper Saddle River, Nueva Jersey. 622 pp. 